Supporting Information for

\title{
The Binding Energy and Bonding in Dialane
}

\author{
By
}

\section{Daniel J. Goebbert, Heriberto Hernandez, Joseph S. Francisco, ${ }^{*}$ and Paul G. Wenthold*}

Contents: Full author list for reference 37, comparison of $\mathrm{AlH}_{4}{ }^{-}$and $\mathrm{CH}_{3} \mathrm{O}^{-}$cross sections, competitive modeling fits and fitting parameters.

Figures $\mathrm{S} 1$ and $\mathrm{S} 2$

Table S1 
Reference 37, full citation:

(37) Frisch, M. J.; Trucks, G. W.; Schlegel, H. B.; G. E. Scuseria; Robb, M. A.; Cheeseman, J. R.; J. A. Montgomery, J.; Vreven, T.; Kudin, K. N.; Burant, J. C.; Millam, J. M.; Iyengar, S. S.; Tomasi, J.; Barone, V.; Mennucci, B.; Cossi, M.; Scalmani, G.; Rega, N.; Petersson, G. A.; Nakatsuji, H.; Hada, M.; Ehara, M.; Toyota, K.; Fukuda, R.; Hasegawa, J.; Ishida, M.; Nakajima, T.; Honda, Y.; Kitao, O.; Nakai, H.; Klene, M.; Li, X.; Knox, J. E.; Hratchian, H. P.; Cross, J. B.; Adamo, C.; Jaramillo, J.; Gomperts, R.; Stratmann, R. E.; Yazyev, O.; Austin, A. J.; Cammi, R.; Pomelli, C.; Ochterski, J. W.; Ayala, P. Y.; Morokuma, K.; Voth, G. A.; Salvador, P.; Dannenberg, J. J.; Zakrzewski, V. G.; Dapprich, S.; Daniels, A. D.; Strain, M. C.; Farkas, O.; Malick, D. K.; Rabuck, A. D.; Raghavachari, K.; Foresman, J. B.; Ortiz, J. V.; Cui, Q.; Baboul, A. G.; Clifford, S.; Cioslowski, J.; Stefanov, B. B.; Liu, G.; Liashenko, A.; Piskorz, P.; Komaromi, I.; Martin, R. L.; Fox, D. J.; Keith, T.; Al-Laham, M. A.; Peng, C. Y.; Nanayakkara, A.; Challacombe, M.; Gill, P. M. W.; Johnson, B.; Chen, W.; Wong, M. W.; Gonzalez, C.; Pople, J. A. Gaussian 03, Revision B.05; Gaussian, Inc.: Pittsburgh, PA, 2003. 


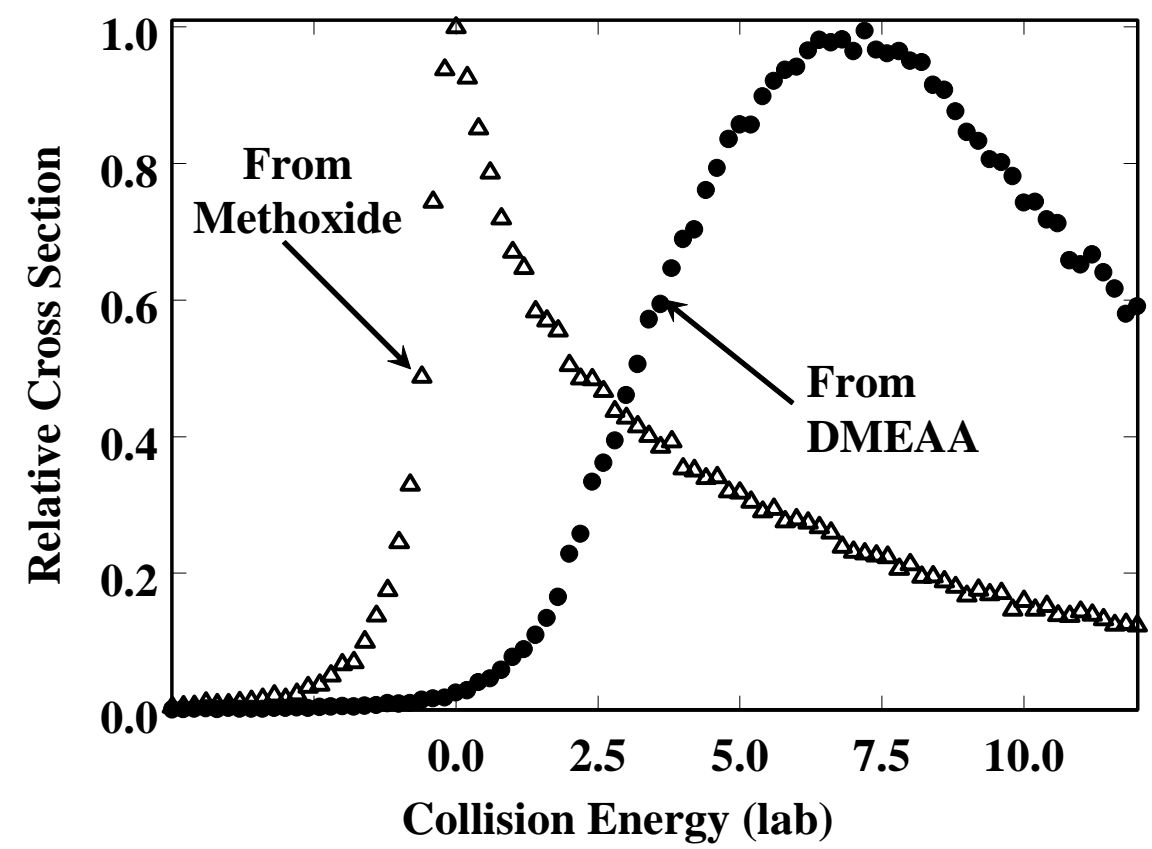

Figure S1. Energy- resolved cross sections for formation of $\mathrm{HCO}_{2}^{-}$in the reaction of the $\mathrm{m} / \mathrm{z} 31$ ion generated from methoxide or dimethylethylaminealane (DMEAA) with $\mathrm{CO}_{2}$.

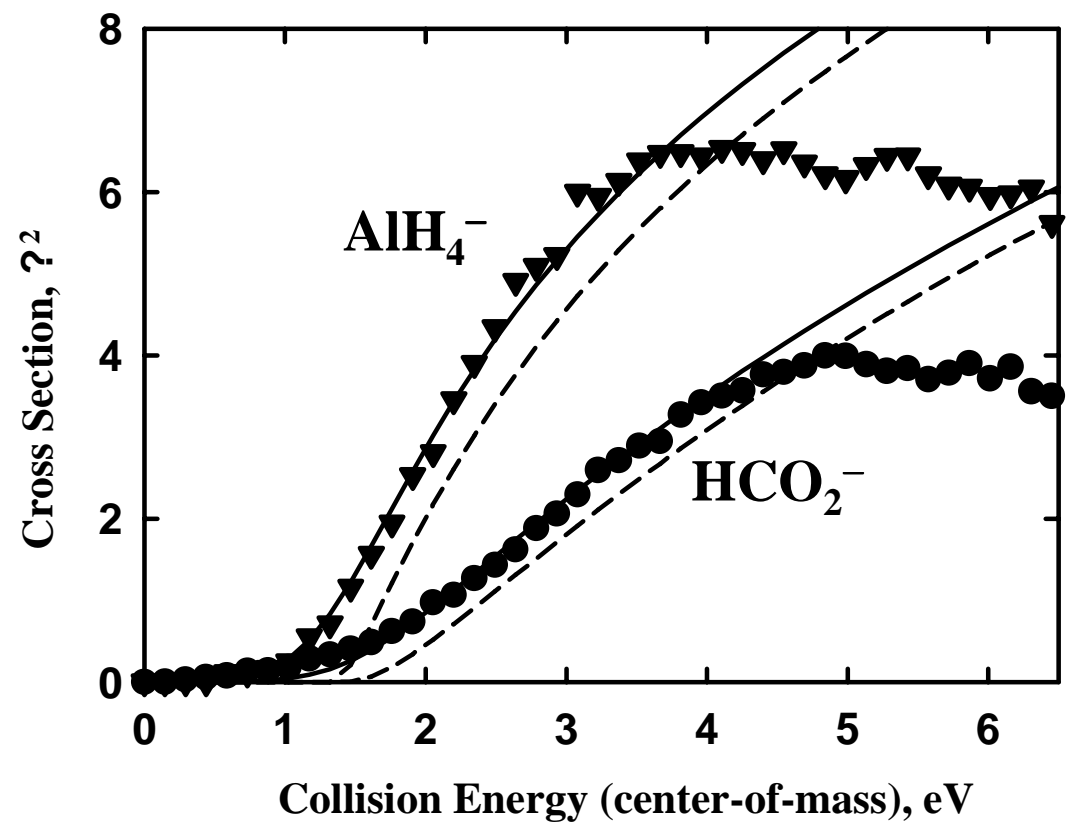

Figure S2. Energy-resolved cross sections for the reaction of $\mathrm{Al}_{2} \mathrm{H}_{7}^{-}$with $\mathrm{CO}_{2}$, along with fits to the data that take into account the competitive reaction processes. 
Table S1. Single Data Set Fitting Parameters of Two Channel Competitive Modeling

\begin{tabular}{ccccccc}
\hline Scaling Factor $^{\mathrm{a}}$ & $\Delta \mathrm{S}^{\ddagger}(1)^{\mathrm{b}}$ & $\Delta \mathrm{S}^{\mathrm{f}}(2)^{\mathrm{b}}$ & $\mathrm{E}_{0}(1)^{\mathrm{c}}$ & $\mathrm{E}_{0}(2)^{\mathrm{c}}$ & $\mathrm{n}$ & $\chi^{2 \mathrm{~d}}$ \\
\hline 0.90 & 1.73 & 3.22 & 1.31 & 1.40 & 1.51 & $6.717 \mathrm{e}-3$ \\
0.95 & 1.71 & 2.42 & 1.33 & 1.40 & 1.45 & $4.773 \mathrm{e}-3$ \\
1.00 & 1.69 & 1.69 & 1.35 & 1.41 & 1.41 & $3.486 \mathrm{e}-3$ \\
1.05 & 1.69 & 0.99 & 1.35 & 1.41 & 1.40 & $3.409 \mathrm{e}-3$ \\
1.10 & 1.69 & 0.36 & 1.34 & 1.37 & 1.40 & $3.489 \mathrm{e}-3$ \\
& & & & & & \\
\hline
\end{tabular}

${ }^{a}$ Scaling factor applied to frequencies of the transition state for channel 2 (hydride transfer)

${ }^{\mathrm{b}}$ Activation entropies for reaction transition states, in cal $/ \mathrm{mol} \cdot \mathrm{K}$

${ }^{\mathrm{c}}$ Dissociation energies obtained from modeling

${ }^{\mathrm{d}} \chi^{2}$ of fit obtained using the corresponding fitting parameters 